\title{
Graphemic and phonemic codings of Chinese characters in short-term retention
}

\author{
LIEN-CHONG MOU and NANCY S. ANDERSON \\ University of Maryland, College Park, Maryland 20742
}

\begin{abstract}
An experiment was conducted to investigate the effect of phonemic or graphemic similarity on recoding of Chinese words. The introduction of acoustic and visual similarity within the list using a retroactive interference design for memory of items showed that both intralist variables were factors causing reduction in recall, with acoustic similarity being more detrimental to short-term retention. An auditory interference task was also shown to have a more severe effect than a visual interference task. Results suggested that dual encoding processes are involved in reading and remembering Chinese.
\end{abstract}

The continuing concern about representation format in memory has led to studies in the problem of differential encoding processes due to orthographical differences (Biederman \& Tsao, 1979; Erickson, Mattingly, \& Turvey, 1972; Park \& Arbuckle, 1977; Tzeng, Hung, \& Wang, 1977; Yik, 1978). Attempts were made to test whether different orthography results in different encoding processes in short-term memory. In alphabetic writing systems such as English, phonetic components in verbal material were found to be important in shortterm memory (Conrad \& Hull, 1964; Rubenstein, Lewis, \& Rubenstein, 1971; Wickelgren, 1965), whereas little evidence has been found to ensure the existence of a visual coding mechanism (Baddeley, 1966; Cimbalo \& Laughery, 1967). However, in dealing with a nonalphabetic writing system such as ideographs, it was postulated that a phonemic transformation is bypassed. Ideographs, being picture-like symbols, may map onto meaning directly (Rozin, Poritsky, \& Sotsky, 1971).

A number of experimental studies have revealed some characteristics of short-term encoding in ideographs. In one study, phonetic encoding was required in reading Japanese Kanji characters, and therefore visually presented linguistic items were phonetically processed regardless of graphemic structure (Erickson et al., 1972). Phonetic components in Chinese characters were also found to play an important role (Tzeng et al., 1977; Treiman, Baron, \& Luk, Note 1; Tzeng \& Hung, Note 2). In one of their experiments, Tzeng et al. (1977) found that the subject's performance was impaired by introducing phonemic similarity into the test materials in a

This research was supported in part by the Biomedical Sciences Support Center of the University of Maryland to the Center for Language and Cognition, and computer time was provided in full by the Computer Science Center. The authors wish to thank David Horton, James Martin, and Carol Mills for critical readings and helpful comments on the manuscript. Requests for reprints should be sent to Nancy S. Anderson, Department of Psychology, University of Maryland, College Park, Maryland 20742. short-term retention task. In more recent studies, Tzeng and Hung (Note 2) showed a left visual field superiority for identification of single Chinese characters but a right visual field superiority effect for making semantic decisions on multiple-character items. These differential effects of Chinese characters on visual information processing suggest a holistic pattern property of ideograms, as well as a phonemic process common to all languages. Therefore, a graphemic property in Chinese could influence a subject's information processing mainly because the characters might be susceptible to visual similarity (Yik, 1978).

The purpose of the present study was to clarify the relative importance of graphemic and phonemic recodings of Chinese ideograms in short-term memory. Chinese ideographs do not have the same sound-to-sight correspondence found in English words or letters and allow different manipulations of phonemic and visual factors. Visual similarity can be introduced by using ideograms with the same radical, resulting in fewer distinguishable features. Phonemic similarity can be introduced for sounds following a procedure similar to that of Tzeng et al. (1977).

\section{METHOD}

\section{Subjects}

Twenty-two Chinese students enrolled in various departments at the University of Maryland served as subjects. They were educated in Taiwan and were considered competent readers of Chinese and fluent speakers of Mandarin. Each subject was paid for his participation in the experiment.

\footnotetext{
Materials and Design

In Mandarin, a Chinese character is a monosyllable, containing one or no consonants before the vowel. All the characters with particular visual and phonemic features were selected from the norms established for frequency of occurrence by Liu and Chuang (1970). The characters were divided into four different classes based on combinations of two pronunciation patterns and two structure features of each word. The four classes are shown in Figure 1.

(1) The pronunciation or phonemic pattern-Based on the
} 


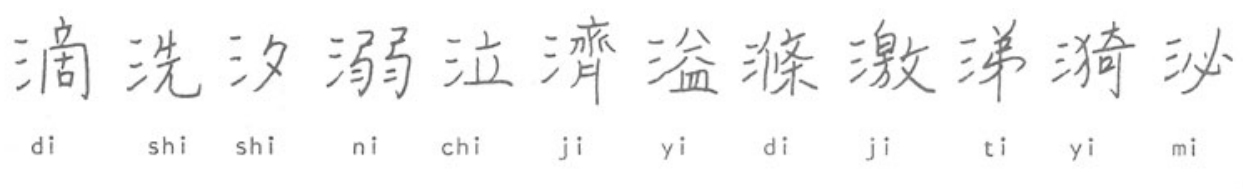

SVDR
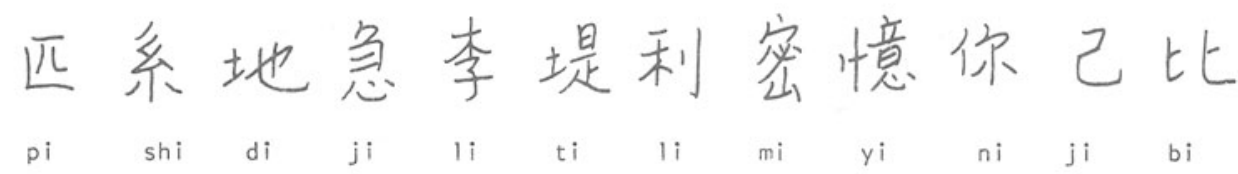

DVSR
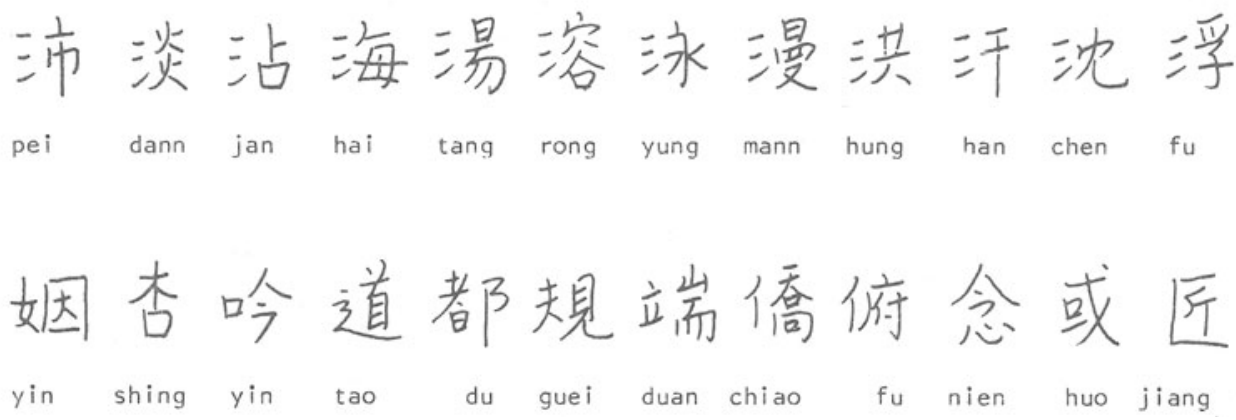

Figure 1. The four sets of Chinese characters with their pronunciations.

similarity or lack of similarity between the vowels, there were two classes in this experiment: (a) same vowel (SV), in which the pronunciation of the characters begins with the same or different consonants but ends with the same vowel and (b) different vowel (DV), in which the pronunciation of the characters begins with the same or different consonants and ends with different vowels. Vowel similarity was selected as a variable rather than consonant similarity because it was found to have more influence on retention by Tzeng et al. (1977).

(2) The structure and visual pattern-The radical in a Chinese word is a part of the whole character and can itself represent a single meaning. Again, two classes were selected: (a) same radical (SR), in which characters have the same radical and thereby contain both graphemic (perceptual) and semantic (conceptual) similarities, and (b) different radical (DR), comprising characters not having the same radical (and having different meanings).

The above two variables were thus combined to yield four different classes: same vowel and same radical (SVSR), same vowel and different radical (SVDR), different vowel and same radical (DVSR), and different vowel and different radical (DVDR). Each of the four classes consisted of 12 different characters. For SR classes, the characters had the same radical meaning, "water"; for SV classes, the characters ended with the same vowel, /i/. Figure 1 gives the details of pronunciation, as well as the four character sets of 12 each.

A retroactive interference paradigm was used in which the target lists were presented visually and the interference lists were presented either visually or orally. Every target list consisted of four different characters sampled exclusively from one of the four classes. The oral interference list consisted of six different characters sampled equally from the SVDR and SVSR lists. Therefore, all six characters in the oral interference list ended with the same vowel, /i/. The visual interference list consisted of six different characters sampled from SVSR and DVSR lists, three from each. Therefore, all six characters in the visual interference list had the same radical, "water." All the variables (pronunciation pattern, structure-feature pattern, and interference tasks) combined to yield a 2 by 2 by 2 factorial design with all subjects tested in each of the eight conditions. There were six lists for each of the eight conditions, yielding 48 total experimental trials for each subject.

\section{Procedure}

Subjects were tested individually, and instructions were given in Mandarin. On each trial, a target list of four characters was presented at a rate of 1 character/sec by slides onto a rearprojection screen using a Kodak Carousel projector. The oral interference task and the visual interference task were administered separately. Half of the subjects were given the oral interference task first and the visual interference second; for the other half, the order was reversed. Subjects wore earphones throughout the experiment. During the oral interference trials, the instructions specified that immediately after the presentation of the last character in the target list, the subjects would hear the signal "repeat." They were to shadow each word in the interference list as it occurred at a 1 -sec rate. Immediately after the subject had finished shadowing the interference list for the second time, a "recall" cue was given and the subject was instructed to write down all four characters of the target list in the correct order within a 15-sec interval; a "stop" signal was given to end the trial. In the visual interference trials, immediately after the target list, a visual display of the interference list was shown at a rate of $2 \mathrm{sec} /$ character. The subject was instructed to copy each of the six characters from the interference list and to count the number of strokes at the same time. Subjects were requested to fill in two columns of six spaces, one for their copy of the interference character, the other for indicating the number of strokes for that character. Immediately after the interference task was finished, the subject was instructed to recall the target list in correct order again during a 15-sec interval. Each trial required $50 \mathrm{sec}$ and the 
intertrial interval was $10 \mathrm{sec}$, with a 5 -min pause given in the middle of the experiment. Two practice trials were given prior to each interference task, and all subjects received a sequence of 24 experimental trials in each task, for a total of 48 trials lasting $1 \mathrm{~h}$. Within each task, the order of the 24 trials was determined by using one of three different randomly generated sequences.

\section{RESULTS}

The mean proportion of correct items averaged over six trials is shown for the eight conditions in Figure 2. Items were considered correct if they occurred in the correct serial position. A three-factor (pronunciation by word structure by interference task) analysis of variance with repeated measures was calculated on the number of errors, and all statistical tests reported were significant at $\mathrm{p}<.05$.

The pronunciation pattern of the target list had a significant main effect on recall $[F(1,21)=56.39$, $\mathrm{MSe}=1.50]$ and indicated that SV lists produced more intralist interference than DV lists did. The word structure feature of the pattern of the target list had a main effect on recall $[\mathrm{F}(1,21)=29.34, \mathrm{MSe}=1.49]$ and showed that characters with the same radical in the target list produced more intralist interference than did characters with different radicals in a recall list. In addition, the type of interference task had a significant main effect $[\mathrm{F}(1,21)=11.11, \mathrm{MSe}=2.92]$ that showed that the oral interference task produced a larger effect on recall than did the visual interference task (correct proportions of .73 and .82 , respectively). There was also a significant interaction between pronunciation pattern and word structure pattern $[F(1,21)=14.69$, $\mathrm{MSe}=2.25$ ], showing that the effect of vowel similarity was more pronounced in the SR condition than in the DR condition (see Figure 2).

In addition, a three-way significant interaction effect was found among the three factors of pronunciation pattern, word structure features of the pattern, and the

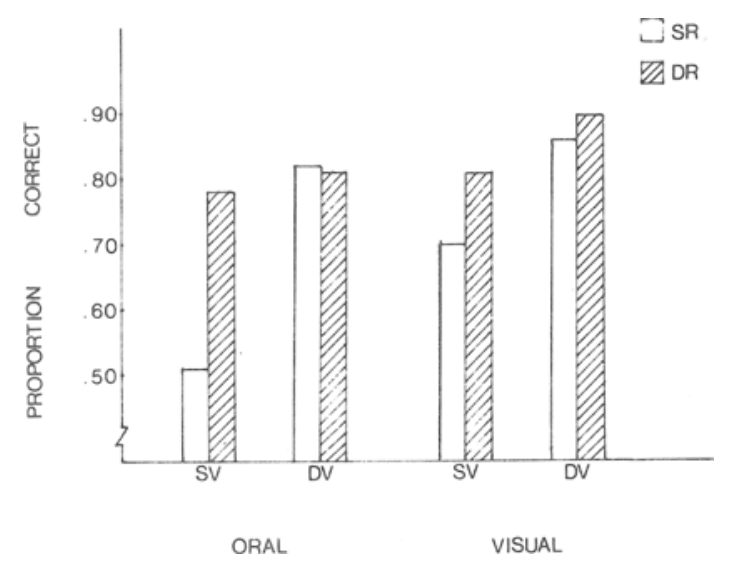

Figure 2. Mean proportions of correct recall in the eight experimental conditions. interference task $[\mathrm{F}(1,21)=15.54, \mathrm{MSe}=2.92]$. As shown in Figure 2, the difference between SR and DR was larger $(27 \%)$ in the oral interference condition than it was in the visual interference condition (10\%).

An analysis of the serial position effect on shortterm retention errors showed that there was an overall primacy effect for all conditions $[F(3,63)=32.75$, $\mathrm{MSe}=.90 \mathrm{]}$. Although the order for oral and visual interference tasks was counterbalanced across subjects, an analysis of these data showed that the order effect was not significant.

\section{DISCUSSION}

In general, phonemic similarity produced retroactive interference on short-term recall, and these results were consistent with the phenomenon found in alphabetic materials (Kintsch \& Buschke, 1969; Wickelgren, 1965). These studies used letters and words, respectively, and found that the vowel similarity in single letters (B, C, D) or homophones in unrelated words caused interference. Thus, regardless of the difference between writing systems, the recoding process is governed in part by the general processes of acoustic coding in working memory (Tzeng et al., 1977).

Although the visual interference task still required the subject to concentrate on writing and counting, the subjects' short-term recall was interfered with less than when they had to shadow vocally during the interval after hearing each item. The task of both writing and counting was not designed to be easier than that of shadowing, and the difference in performance can best be interpreted in terms of reducing capacity or memory load from interference in the same modality. If the characters were not recoded in an acoustic form, the oral interference task should not necessarily have had a more detrimental effect than the visual interference task.

Evidence was found that both acoustic and visual encodings were used. The distinctive word features combined with phonemic dissimilarity (DRDV) resulted in better performance than did the distinctive word features combined with phoneme similarity (DRSV). However, in all comparisons shown in Figure 2, distinctive word features resulted in the best performance (almost equal performance in the case of DRDV and SRDV with oral interference). These results are consistent with the result obtained by Yik (1978), who showed a significant acoustic encoding and a significant visual similarity effect in the recall of Chinese words. Support for visual encoding has also been reported by Biederman and Tsao (1979). This result is different from that using English materials, in which recall was not found to be affected by visual similarity (Baddeley, 1966). It appears that the present subjects could make use of either the distinctiveness of the graphemic cue or the phonemic cue to improve their memory performance, and the present results provide further evidence for dual encoding in recalling Chinese characters with visual features different from those found in English.

\section{REFERENCE NOTES}

1. Treiman, R. A., Baron, J., \& Luk, K. Type of orthography affects use of sound in silent reading. Paper presented at the meeting of the Eastern Psychological Association, Philadelphia, Penn., 1979.

2. Tzeng, O. J. L., \& Hung, D. L. Reading in the nonalphabetic writing system: Some experimental studies. Paper presented at the Cross-Language Conference on Orthography, Reading, and Dyslexia, Bethesda, Md., 1978. 


\section{REFERENCES}

BAdDeley, A. D. Short term memory for word sequences as a function of acoustic, semantic, and formal similarity. Quarterly Journal of Experimental Psychology, 1966, 18, 362-365.

Biederman, I., \& Tsao, Y. On processing Chinese ideographs and English words: Some implications from Stroop-test results. Cognitive Psychology, 1979, 11, 125-132.

Cimbalo, R. S., \& Laughery, K. B. Short term memory: Effects of auditory and visual similarity. Psychonomic Science, 1967, 8, 57-58.

Conrad, R., \& Hull, A. J. Information, acoustic confusion and memory span. British Journal of Psychology, 1964, 55, 429-432.

Erickson, D., Mattingly, I. G., \& Turvey, M. T. Phonetic coding of Kanji. Journal of the Acoustic Society of America, 1972, 52, 133.

KintsCH, W., \& Buschкe, H. Monophones and synonyms in short-term memory. Journal of Experimental Psychology, 1969, 80, 403-407.

Liv, I. M., \& Chuang, C. J. [Scaling 1,200 Chinese characters for meaningfulness] (in Chinese). Acta Psychologic Taiwanica, $1970,12,33-52$.
Park, S., \& Arbuckle, T. Y. Ideograms versus alphabets: Effects of script on memory in "biscriptual" Korean subjects. Journal of Experimental Psychology: Human Learning and Memory, 1977, 3, 631-642.

Rozin, P., Poritsky, S., \& Sotsky, R. American children with reading problems can easily learn to read English represented by Chinese characters. Science, 1971, 171, 1264-1267.

Rubenstein, H., Lewis, S. S., \& Rubenstein, M. A. Evidence for phonetic recoding in visual word-recognition. Journal of Verbal Learning and Verbal Behavior, 1971, 10, 645-657.

Tzeng, O. J. L., HunG, D. L., \& WANG, W. S. Y. Speech recoding in reading Chinese characters. Journal of Experimental Psychology: Human Learning and Memory, 1977, 3, 621-630.

WiCKelgReN, W. A. Short-term memory for phonemically similar lists. American Journal of Psychology, 1965, 78, 567-574.

YIK, W. F. The effect of visual and acoustic similarity on shortterm memory for Chinese words. Quarterly Journal of Experimental Psychology, 1978, 30, 487-494.

(Received for publication May 22, 1981.) 